\title{
Treatment of 85 Cases with Chronic Rhinitis by Acupuncture
}

\author{
AN Hua (安华) \\ Qinhuangdao Port Hospital, Hebei 066000, P. R. China
}

\author{
【关键词】针炎疗法; 艾条炎; 鼻炎 \\ 【Key Words】 Acupuncture-moxibustion Therapy; Moxa Stick Moxibustion; Rhinitis \\ 【CLC Number】 R246.8 \\ 【Document Code】 B
}

Patients with chronic rhinitis may not respond well to medications. The author treated 85 cases using acupuncture. The result is reported as follows.

\section{General Data}

Of 85 cases, there were 61 males and 24 females, and 79 cases had been treated with medications.

\section{Treatment Method}

Major points: Fengchi (GB 20), Hegu (LI 4) and Zusanli (ST 36).

Modifications: Added Fengmen (BL 12) and Feishu (BL 13) for wind-cold; applied bloodletting to Yintang (Ex-HN 3) for wind-heat; applied moxibustion to Dazhui (GV 14) for deficiencycold.

Method: Puncture perpendicularly using filiform needles of $0.35 \mathrm{~mm}$ in diameter and $40-50 \mathrm{~mm}$ in length, followed by reducing manipulation (reinforcing manipulation for the pattern of deficiency-cold). For moxibustion, apply birdpecking moxibustion until a hot sensation occurs. For bloodletting, prick using a three-edged needle to allow a couple of blood drops spontaneously or to squeeze drops of blood.

The treatment was conducted once a day, 15 times as a course of treatment.

\section{Treatment Result}

\subsection{Therapeutic effect criteria}

Recovery: Absence of clinical manifestations with normal findings by rhinoscopy.

Marked effect: Substantial improvement of stuffy or runny nose with significant alleviation of nasal congestion by rhinoscopy.

Failure: No improvement in clinical manifesttations or rhinoscopy findings.

\subsection{Treatment result}

After two courses of treatment, 61 cases obtained recovery, 21 cases obtained a marked effect and 3 cases had no effect. The total effective rate was $96.5 \%$.

\subsection{Case study}

A 51-year-old male patient had had stuffy and runny nose for 6 years. Other symptoms included olfaction disorder, yellow or occasionally bloodstripped nasal discharges, dry nose, dry stools and scanty, dark-yellow urine. The tongue was slightly red with a thin, yellow coating. The pulse was slippery and rapid. The patient was, therefore, diagnosed with chronic simple rhinitis due to windheat affecting the lung. Fengchi (GB 20), Hegu (LI 4) and Zusanli (ST 36) were punctured with reducing manipulation (20 min), coupled with bloodletting on Yintang (Ex-HN 3). The treatment was conducted once a day, 17 times in total. After that, the patient showed a complete recovery with normal rhinoscopy findings. The two-year followup didn't show any relapses.

\section{Remark}

Traditional Chinese medicine holds that chronic rhinitis is due to wind-cold or wind-heat obstructing lung-qi. Fengchi (GB 20) can dispel wind. Hegu (LI 4) acts to tonify lung-qi and dissipate cold. As the Earth point, Zusanli (ST 36) acts to tonify the lung by strengthening the spleen, and it can also strengthen the immune system. For wind-heat, bloodletting acts to clear heat. For deficiency-cold, moxibustion acts to warm yang and strengthen the bodily resistance.

Translator: HAN Chou-ping (韩丑萍)

Received Date: June 10, 2010 